Tema: Aciaria Oxigênio

\title{
INFLUÊNCIA DAS FASES PRESENTES NA DESFOSFORAÇÃO DE FERRO-GUSA*
}

\author{
Felipe Costa Broseghini ${ }^{1}$ \\ Sábata Marla Reis Durães Oliveira ${ }^{1}$ \\ Raphael de Alcântara Sampaio ${ }^{1}$ \\ Elsomar Biancardi Guimarães de Oliveira ${ }^{2}$ \\ José Roberto de Oliveira ${ }^{3}$
}

\section{Resumo}

O objetivo deste trabalho é estudar a influência das fases sólidas e líquidas formadas na desfosforação de ferro gusa. As escórias foram feitas a bases de Cal, resíduo de mármore e carepa. Neste trabalho também foi investigada a utilização de fluorita e barrilha nas escórias produzidas, objetivando avaliar as melhores condições para o processo de desfosforação de ferro-gusa. Experimentos em escala laboratorial foram realizados com a adição das misturas desfosforantes iniciais, na forma in natura, a temperatura de $1.400^{\circ} \mathrm{C}$ e de composição química conhecida. Para efeitos comparativos das adições, os experimentos foram realizados com agitação mecânica. Foram utilizados cadinhos de MgO para a fusão do ferro-gusa. Amostras do ferro-gusa foram retiradas em intervalos apropriados, para 0 monitoramento de sua composição química e, após o tratamento, foram obtidas amostras da escória final, sendo comparadas as composições finais as iniciais. Os resultados foram comparados com vista na análise da eficiência das escórias e no processo de desfosforação, com o uso dos programas ThermoCalc e FactSage.

Palavras-chave: Desfosforação; Ferro-gusa; ThermoCalc; FactSage.

\section{INFLUENCE OF PHASE COMPOSITION IN HOT METAL DEPHOSPHORIZATION}

\begin{abstract}
The aim of this work is to study the influence of solid and liquid phase composition in hot metal dephosphorization. The slag was composed by lime, marble waste and mill scale. This work also investigated the use of fluorite and soda ash, to evaluate the best conditions for the process of hot metal dephosphorization. Laboratory scale experiments were conducted with the addition of the initial mix, in natura, in hot metal at a temperature of $1,400^{\circ} \mathrm{C}$ and known chemical composition. For comparative purposes, the experiments were carried out with mechanical stirring. MgO crucibles were used for melting. Samples of hot metal were taken at appropriate intervals, to monitor its chemical composition and, after the treatment, the final slag samples were obtained and compared the final compositions with the initials. The results were compared in order to analyze the efficiency of the slag and the dephosphorization process, with the use of Thermocalc and FactSage software.
\end{abstract}

Key words: Dephosphorization; Hot metal; ThermoCalc; FactSage.

1 Mestrando, Programa de Pós-Graduação em Engenharia Metalúrgica e de Materiais, IFES, Espírito Santo, Vitória, Brasil.

2 Mestre em Engenharia Metalúrgica e Materiais, Engenheiro Metalurgista, Villares Metals S.A., Sumaré, São Paulo, Brasil.

3 Engenheiro Metalurgista, Professor Doutor, Programa de Pós-Graduação em Engenharia Metalúrgica e Materiais, IFES, Espírito Santo, Vitória, Brasil.

\footnotetext{
* Contribuição técnica ao 450 Seminário de Aciaria - Internacional, 25 a 28 de maio de 2014,
} 


\section{INTRODUÇÃO}

A reação de desfosforação, pode ser representada pelas Equações 1 e 2 [1].

$$
\begin{aligned}
& 2 \underline{P}+5(\mathrm{FeO})_{(l)}+3(\mathrm{CaO})_{(s)}=\left(3 \mathrm{CaO} \cdot P_{2} \mathrm{O}_{5}\right)_{(s)}+5 \underline{F e} \\
& \Delta G^{0}=-204.450+83,55 . T(\mathrm{~J} / \mathrm{mol}) \\
& 2 \underline{P}+5 / 2 O_{2(g)}+3(\mathrm{CaO})_{(s)}=\left(3 \mathrm{CaO} \cdot P_{2} O_{5}\right)_{(s)} \\
& \Delta G^{0}=-499.190+154,16 . T(\mathrm{~J} / \mathrm{mol})
\end{aligned}
$$

Os principais fatores que influenciam o equilíbrio da reação de desfosforação são: temperatura, potencial de oxigênio, atividade do $\mathrm{CaO}$ na escória e a presença de elementos que aumentam a atividade henriana do fósforo no ferro-gusa líquido.

Segundo Campos [2], admitindo-se que o processo de desfosforação ocorra através da reação 2, na interface metal/escória, a formação do composto $3 \mathrm{CaO} \cdot \mathrm{P}_{2} \mathrm{O}_{5}$ é responsável por reduzir a atividade do $\mathrm{P}_{2} \mathrm{O}_{5}$ na escória:

Tomando-se os estados padrões e considerando o sistema em equilíbrio, obtém-se a Equação 3, a partir do equilíbrio químico, para predizer o teor de fósforo final:

$$
\ln [\% P]=\frac{1}{2} \ln a_{\left(3 \mathrm{CaO} . \mathrm{P}_{2} \mathrm{O}_{5}\right)}+\frac{\Delta G^{0}}{2 R T}-\frac{5 \mu_{\mathrm{O}_{2}}}{4 R T}-\frac{3}{2} \ln a_{(\mathrm{CaO})}-\ln f_{P}
$$

A Equação 5 é útil para verificar os fatores termodinâmicos que influenciam na reação de desfosforação, como temperatura, potencial de oxigênio, $a_{\mathrm{CaO}}, a_{3 \mathrm{CaOP} 205} \mathrm{e}$ o coeficiente de atividade henriano do fósforo.

Segundo Borode [3], uma medida da eficiência do processo de desfosforação é o coeficiente de partição real do fósforo ( $\left.L_{P}^{\text {real }}\right)$, entre a escória e o metal líquido, calculado para uma dada condição, sendo definida pela Equação 4:

$$
L_{P}^{\text {real }}=(\% P) /[\% P]
$$

Onde: $(\% P)=$ porcentagem em peso de fósforo dissolvido na escória e $[\% P]=$ porcentagem em peso de fósforo dissolvido no metal líquido.

Suito e Inoue [4] realizaram experimentos de desfosforação, onde escória do sistema CaO-FeO- $\mathrm{P}_{2} \mathrm{O}_{5}$ foi fundida em um cadinho de ferro sob atmosfera de argônio, a temperatura de $1400^{\circ} \mathrm{C}$. A partir dos resultados, os autores desenvolveram a equação 5 para estimar o $L_{P}{ }^{\text {equilbrio }}$ na interface entre metal/escória:

$$
\log \frac{(\% P)}{[\% P]}=0,072\{(\% C a O)+0,3 .(\% M g O)\}+2,5 \cdot \log \left(\% . F e_{\text {total }}\right)+\frac{11570}{T}-10,52
$$

Os valores para o $L_{P}{ }^{\text {equilibrio }}$, encontrados pelo modelo proposto, variaram de $10^{2}$ a $10^{4}$ para uma faixa de temperatura entre $1400-1600^{\circ} \mathrm{C}$.

O modelo de Healy [5], com base na teoria iônica das escórias, descreve $L_{P}{ }^{\text {equilibrio }}$, para sistema metal/escória, contendo baixas quantidades de $\mathrm{MnO}, \mathrm{MgO}$ e $\mathrm{Al}_{2} \mathrm{O}_{3}$, como mostra a Equação 6:

$$
\ln \frac{(\% P)}{[\% P]}=\frac{22350}{T}+0,08 .(\% C a O)+2,5 \log \left(F e_{\text {total }}\right)-16
$$

* Contribuição técnica ao 45 Seminário de Aciaria - Internacional, 25 a 28 de maio de 2014, 
A correlação é válida para concentrações de CaO na escória menores que 50\%. O valor do coeficiente de partição calculado através do modelo de Healy [5] varia entre $10^{2}$ e $10^{4}$ para uma faixa de temperatura entre $1400-1600^{\circ} \mathrm{C}$.

A capacidade de fosfato é uma medida da capacidade de desfosforação da escória, sob um dado potencial de oxigênio, estando relacionada com a atividade dos íons de oxigênio na escória [6]. Young [7] selecionou dados reportados na literatura referentes à partição do fósforo entre ferro-gusa e escória, na qual propôs a Equação 7 para o cálculo da capacidade de fosfato $\left(C_{P}\right)$ de escórias do sistema $\mathrm{CaO}-\mathrm{FeO}-\mathrm{SiO}_{2}-\mathrm{MnO}-\mathrm{P}_{2} \mathrm{O}_{5}$, e os valores encontrados, a partir dos dados da literatura coletados pelo autor, variaram de $10^{2}$ a $10^{7}$.

$$
\begin{aligned}
& \log C_{P}=-18,184+35,84 \Lambda-22,35 \Lambda^{2}+\frac{22930 \Lambda}{T}-0,06257(\% \mathrm{FeO}) \\
& -0,04256(\% \mathrm{MnO})+0,359\left(\% P_{2} \mathrm{O}_{5}\right)^{0.3}
\end{aligned}
$$

Diferentes processos de desfosforação do ferro-gusa acontecem no carro-torpedo e/ou na panela. A desfosforação em carro-torpedo pode ser realizado com injeção profunda no interior do ferro-gusa líquido, através de lança, de misturas a base de $\mathrm{CaO}, \mathrm{FeO}, \mathrm{CaF}_{2}$ e $\mathrm{Na}_{2} \mathrm{CO}_{3}$ [8]. Também se pode usar a injeção de oxigênio com $\mathrm{o}$ FeO, para aumentar a taxa de desfosforação.

O processo de desfosforação em panela de ferro-gusa consiste em um equipamento de injeção, através de uma lança de imersão utilizando o gás nitrogênio $\left(\mathrm{N}_{2}\right)$, para adição de pós como agente desfosforante [9].

A Nippon Steel desenvolveu o refino de ferro-gusa chamado de processo "MURC" (Multirefining Converter), em que os processos de desfosforação e de descarburação são realizados continuamente no mesmo convertedor LD. Este processo é mostrado esquematicamente na Figura 1 [10].

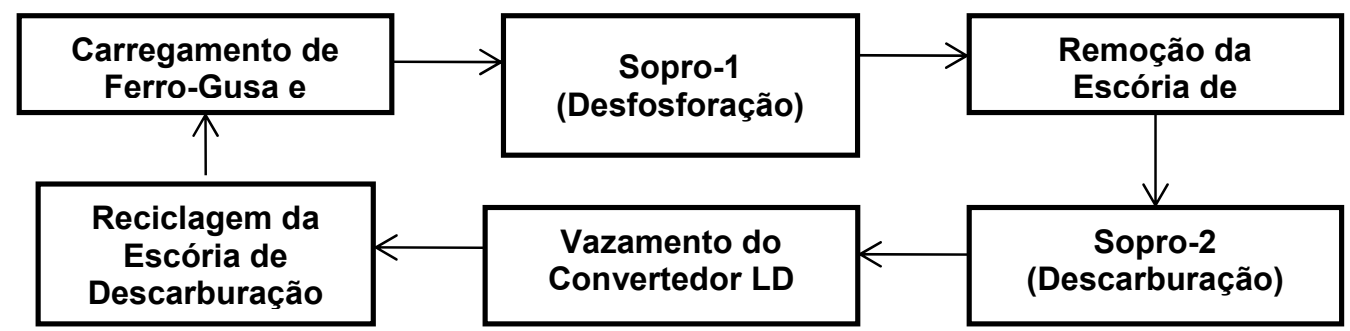

Figura 1. Etapas do processo MURC da Nippon Steel [10].

Conforme mostrado na Figura 1, a etapa do primeiro sopro consiste para ocorrer o processo de desfosforação. Após, é realizada a imediata remoção de escória, dando início à segunda etapa do sopro com o processo de descarburação. Após o vazamento do aço, a escória formada na descarburação é reciclada no processo de desfosforação. Este procedimento permite a diminuição de $50 \%$ do volume de escória gerada, se comparado como processo convencional, além da redução do tempo de processo.

Para reduzir o teor do fósforo no ferro-gusa, o processo de desfosforação tem sido realizado com escórias multifásicas do sistema $\mathrm{CaO}-\mathrm{FeO}-\mathrm{SiO}_{2}-\mathrm{P}_{2} \mathrm{O}_{5}$, sendo realizado na região saturada com silicato dicálcio $\left(2 \mathrm{CaO} . \mathrm{SiO}_{2}\right)$ [11]. Em muitos casos, a escória está coexistindo em um estado sólido-líquido, contendo o composto $2 \mathrm{CaO} . \mathrm{SiO}_{2}$, como a principal fase sólida [12].

É importante entender o papel das fases sólidas na desfosforação. Essas fases formam o $2 \mathrm{CaO} . \mathrm{SiO}_{2}-3 \mathrm{CaO} . \mathrm{P}_{2} \mathrm{O}_{5}$ que fixa $0 \mathrm{P}_{2} \mathrm{O}_{5}$ como $3 \mathrm{CaO} . \mathrm{P}_{2} \mathrm{O}_{5}$, principal

* Contribuição técnica ao $45^{\circ}$ Seminário de Aciaria - Internacional, 25 a 28 de maio de 2014, 
produto da desfosforação, dentro da temperatura de tratamento e ao longo de uma ampla faixa de composição química da escória. Porém, no processo industrial, para minimizar o desgaste de refratário dos fornos, durante o refino do ferro-gusa, a escória está coexistindo em um estado sólido-líquido, sendo que está saturada em $\mathrm{MgO}$, o que reduz o desgaste de refratários e, consequentemente, sua capacidade de desfosforar. Dessa forma, maiores quantidades de $\mathrm{CaO}$ são adicionadas para forçar o processo de desfosforação do ferro-gusa líquido [13], minimizando o aumento da solubilidade do MgO [14] nas escórias.

\section{MATERIAIS E MÉTODOS}

As quantidades das misturas desfosforantes foram calculadas via reação de dessiliciação e desfosforação, sabendo-se que todas as amostras de ferro-gusa possuem massa constante de 800 gramas e teores iniciais de silício e de fósforo: 0,32 e $0,11 \%$, respectivamente. A Tabela 1 apresenta um resumo das misturas desfosforantes que foram preparadas para a realização dos experimentos de desfosforação com agitação mecânica:

Tabela 1. Misturas desfosforantes preparadas para os experimentos

\begin{tabular}{|ccc|}
\hline$N^{\circ}$ das Misturas & Fonte de CaO & Misturas Desfosforantes \\
\hline $\mathbf{1}$ & & Cal + Carepa \\
$\mathbf{2}$ & $\mathrm{Cal}$ & $\mathrm{Cal}+\mathrm{Carepa}+\mathrm{CaF}_{2}$ \\
$\mathbf{3}$ & & $\mathrm{Cal}+\mathrm{Carepa}+\mathrm{Na}_{2} \mathrm{CO}_{3}$ \\
$\mathbf{4}$ & & $\mathrm{Cal}+\mathrm{Carepa}+\mathrm{CaF} \mathrm{Na}_{2} \mathrm{CO}_{3}$ \\
$\mathbf{5}$ & & Resíduo de mármore $+\mathrm{Carepa}$ \\
$\mathbf{6}$ & Mármore & Resíduo de mármore $+\mathrm{Carepa}+\mathrm{CaF}_{2}$ \\
$\mathbf{7}$ & & Resíduo de mármore + Carepa $+\mathrm{Na}_{2} \mathrm{CO}_{3}$ \\
$\mathbf{8}$ & & Resíduo de mármore + Carepa $+\mathrm{CaF}_{2}+\mathrm{Na}_{2} \mathrm{CO}_{3}$ \\
\hline
\end{tabular}

A Tabela 2 mostra como ficou a composição química inicial das misturas desfosforantes utilizadas nos experimentos de desfosforação.

Tabela 2. Composição química inicial das misturas desfosforantes

\begin{tabular}{|c|c|c|c|c|c|c|c|c|c|}
\hline \multirow{2}{*}{$\begin{array}{l}\text { No Mist. } \\
\text { Desf. }\end{array}$} & \multicolumn{6}{|c|}{ Composição química (\% em massa) } & \multirow{2}{*}{$\begin{array}{l}\text { Massa } \\
\mathrm{CaO}(\mathrm{g})\end{array}$} & \multirow{2}{*}{\begin{tabular}{|c|} 
Massa \\
$\mathrm{FeO}(\mathbf{g})$
\end{tabular}} & \multirow{2}{*}{$\mid \begin{array}{c}\text { Massa } \\
\text { Total (g) }\end{array}$} \\
\hline & |CaO & $\mathrm{FeO}$ & $\left|\mathrm{SiO}_{2}\right|$ & $\left|\mathrm{Na}_{2} \mathrm{O}\right|$ & $\left|\mathbf{C a F}_{2}\right|$ & | MgO & & & \\
\hline 1 & 23,0 & 74,5 & 2,5 & 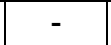 & - & - & 15,6 & 50,6 & 67,9 \\
\hline 2 & 22 & & & - & 2,9 & - & & & \\
\hline 3 & 22, & 72 & 2 & $2, \varepsilon$ & & - & & & \\
\hline 4 & 21,7 & 70,3 & 2 & 2,8 & 2,8 & 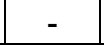 & 15 & 50,6 & 71,9 \\
\hline 5 & 21,2 & 68,6 & 2,5 & & & 7,7 & 15,6 & 50,6 & 73,6 \\
\hline 6 & 20 & & & - & 2,9 & 7 & & & \\
\hline 7 & 20, & 66 & 2 , & 2,8 & 1 & 7,5 & 15 & 50 & 75,8 \\
\hline 8 & 20,0 & 64,8 & 2,3 & 2,8 & 2,8 & 7,2 & 15,6 & 50,6 & 78,0 \\
\hline
\end{tabular}

Como proposta, considerou-se manter constante a mesma massa de $\mathrm{CaO}$ e de FeO utilizada em todas as misturas desfosforantes. A presença de $\mathrm{MgO}$ na composição química das misturas desfosforantes é proveniente do resíduo de mármore.

Cálculos termodinâmicos empíricos foram realizados, através de modelos presentes na literatura, para determinar os seguintes parâmetros: coeficiente de atividade do $\mathrm{P}_{2} \mathrm{O}_{5}$, basicidade ótica, capacidade de fosfato e o coeficiente de partição de equilíbrio do fósforo.

As simulações termodinâmicas foram realizadas através dos softwares de termodinâmica computacional: ThermoCalc e FactSage, utilizados para a

* Contribuição técnica ao $45^{\circ}$ Seminário de Aciaria - Internacional, 25 a 28 de maio de 2014, 
determinação das condições de equilíbrio da reação de desfosforação, bem como caracterizar as escórias finais quanto a porcentagem de líquido e sólido formado, os valores de atividade dos óxidos e porcentagem de fases formadas.

\section{RESULTADOS E DISCUSSÃO}

Amostras de escória, após o final do processo de desfosforação, foram retiradas e analisadas. A Tabela 3 resume a composição química final das escórias desfosforantes obtidas.

Tabela 3. Análise química das escórias finais para os experimentos realizados com cadinho de MgO-C e impeller de alumina

\begin{tabular}{|ccccccccc|}
\hline Compostos & \multicolumn{8}{c|}{ \% em massa dos óxidos na Escória } \\
\hline Experimento $\mathbf{N}^{\circ}$ & $\mathbf{1}$ & $\mathbf{2}$ & $\mathbf{3}$ & $\mathbf{4}$ & $\mathbf{5}$ & $\mathbf{6}$ & $\mathbf{7}$ & $\mathbf{8}$ \\
\hline $\mathbf{C a O}$ & 47,04 & 32,34 & 32,50 & 30,92 & 43,17 & 34,12 & 37,63 & 24,11 \\
$\mathbf{F e O}$ & 14,53 & 1,48 & 1,75 & 2,06 & 15,68 & 12,03 & 14,32 & 19,06 \\
$\mathrm{SiO}_{\mathbf{2}}$ & 27,03 & 27,84 & 31,80 & 29,36 & 20,04 & 18,16 & 20,31 & 17,05 \\
$\mathbf{A l}_{\mathbf{2}} \mathbf{O}_{\mathbf{3}}$ & 2,18 & 12,16 & 14,25 & 12,37 & 4,16 & 9,18 & 9,00 & 13,07 \\
$\mathbf{M g O}$ & 1,49 & 21,23 & 15,80 & 17,41 & 9,52 & 18,41 & 10,07 & 17,90 \\
$\mathbf{M n O}$ & 5,18 & 0,84 & 0,84 & 1,68 & 5,22 & 3,61 & 3,03 & 2,20 \\
$\mathbf{P}_{\mathbf{2}} \mathbf{O}_{\mathbf{5}}$ & 2,54 & 0,32 & 0,09 & 0,22 & 2,20 & 1,16 & 1,46 & 0,69 \\
$\mathbf{C a F}_{\mathbf{2}}$ & 0,00 & 3,78 & 0,00 & 3,01 & 0,00 & 3,22 & 0,00 & 2,32 \\
$\mathbf{N a}_{\mathbf{2}} \mathbf{O}$ & 0,01 & 0,01 & 2,97 & 2,97 & 0,01 & 0,01 & 4,18 & 3,54 \\
\hline
\end{tabular}

Conforme observado na Tabela 3, a composição química inicial foi alterada. Provavelmente após os primeiros 5 minutos da adição das misturas desfosforantes iniciais sobre a superfície do ferro-gusa líquido, a temperatura de $1400^{\circ} \mathrm{C}$, a composição química das misturas desfosforantes iniciais foi alterada, devido a contaminação com $\mathrm{Al}_{2} \mathrm{O}_{3}$ e $\mathrm{MgO}$, deixando de ser escórias do sistema CaO-FeO$\mathrm{SiO}_{2}$, exceto para as escórias $\mathrm{N}^{0} 1$ (Cal + Carepa) e $\mathrm{N}^{\mathrm{O}} 5$ (RM + Carepa). Nos primeiros minutos dos experimentos que utilizaram fluorita $\left(\mathrm{CaF}_{2}\right)$ e barrilha $\left(\mathrm{Na}_{2} \mathrm{CO}_{3}\right)$, houve o desgaste do cadinho de $\mathrm{MgO}-\mathrm{C}$ e do impeller de $\mathrm{Al}_{2} \mathrm{O}_{3}$.

Portanto, os resultados serão discutidos com bases nas escórias finais e não nas iniciais.

A Figura 2 mostra a evolução do teor de fósforo, conforme os resultados.

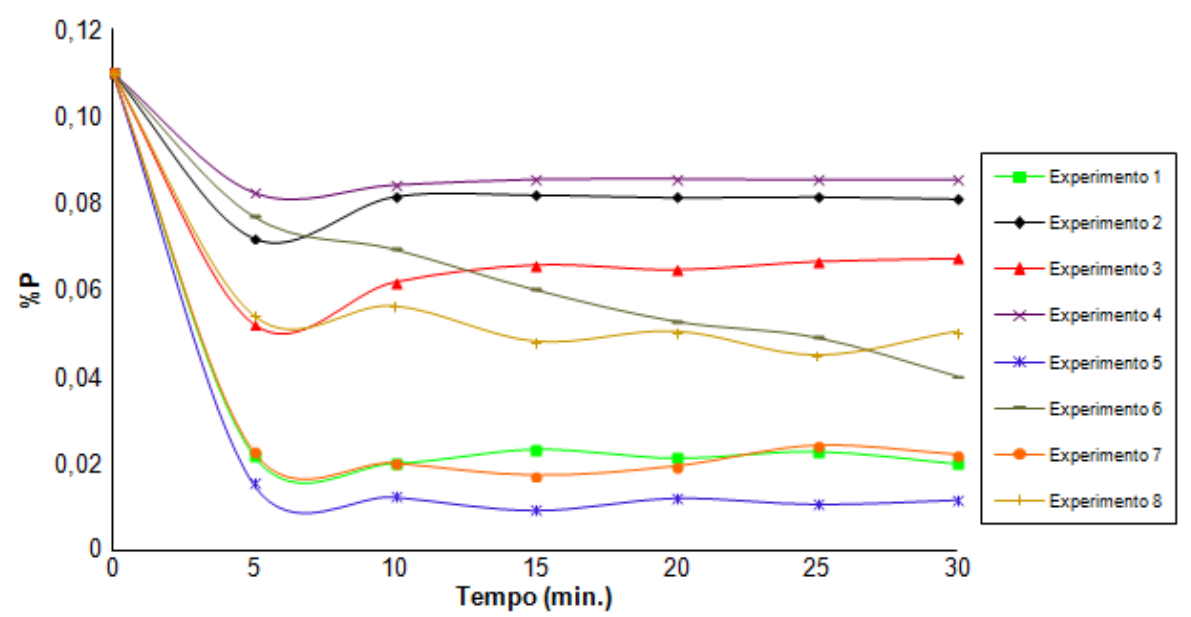

Figura 2. Evolução do teor de fósforo do ferro-gusa em relação ao tempo de processo.

* Contribuição técnica ao $45^{\circ}$ Seminário de Aciaria - Internacional, 25 a 28 de maio de 2014, 
Conforme observado na Figura 2, o teor de fósforo foi reduzido no ferro-gusa líquido, durante os primeiros 5 minutos de tratamento, em todos os experimentos. A Figura 3 mostra o rendimento da desfosforação, (\%)De-P, no final dos experimentos. Onde o rendimento é dado pela Equação 8.

$(\%) D e-P=\frac{[\% P]_{\text {inicial }}-[\% P]_{\text {final }}}{[\% P]_{\text {inicial }}} \times 100$

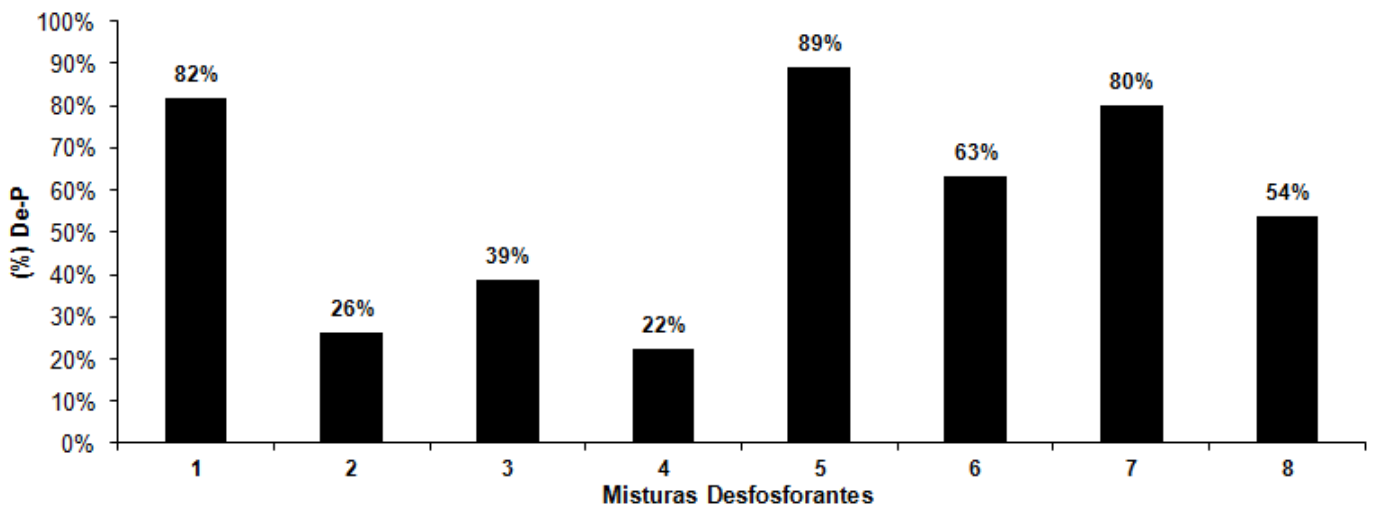

Figura 3. Rendimento da desfosforação do ferro-gusa com o uso das misturas desfosforantes iniciais.

Conforme observado na Figura 3, a mistura desfosforante 5 (Resíduo de Mármore + Carepa) obteve o maior rendimento no processo de desfosforação do ferro-gusa, com $89 \%$, chegando com o teor final de 0,0118\% de fósforo. Esse resultado mostra possibilidade de utilização de misturas desfosforantes a base de resíduo de mármore, com elevado teor de FeO, sem a utilização de materiais fundentes: fluorita $\left(\mathrm{CaF}_{2}\right)$ e barrilha $\left(\mathrm{Na}_{2} \mathrm{CO}_{3}\right)$.

$\mathrm{Na}$ Tabela 4 o número das misturas estão ordenados em função do teor do $\mathrm{P}_{\text {eq, }}$, calculado pelo programa FactSage, entre a composição inicial do ferro-gusa líquido e as misturas desfosforantes iniciais.

Tabela 4. Temperatura de fusão, \% de líquido e \% de sólido das misturas desfosforantes iniciais com relação ao fósforo de equilíbrio

\begin{tabular}{|c|c|c|c|c|c|c|c|}
\hline \multirow{2}{*}{$\begin{array}{l}\mathrm{N}^{\circ} \\
\text { Mistura }\end{array}$} & \multirow{2}{*}{$\begin{array}{l}\text { Temp. } \\
\text { Exper. }\left({ }^{\circ} \mathrm{C}\right)\end{array}$} & \multirow{2}{*}{$\begin{array}{l}\text { Temp. } \\
\left.\text { Fusão ( }{ }^{\circ} \mathrm{C}\right)\end{array}$} & \multirow{2}{*}{$\begin{array}{l}\text { (\%) } \\
\text { Líq. }\end{array}$} & \multirow{2}{*}{$\begin{array}{l}\text { (\%) } \\
\text { Sól. }\end{array}$} & \multicolumn{2}{|c|}{ (\%) Sólido } & \multirow{2}{*}{$\% P_{\text {eq. }}$} \\
\hline & & & & & $\mathrm{NaF}$ & $\mathrm{MgO}$ & \\
\hline 8 & 1400 & 1747 & 94,36 & 5,64 & 5,76 & 94,24 & 0,000053 \\
\hline 4 & 1400 & 1264 & 100 & - & - & - & 0,000058 \\
\hline 7 & 1400 & 1763 & 94,59 & 5,41 & - & 100 & 0,000061 \\
\hline 3 & 1400 & 1308 & 100 & - & - & - & 0,000067 \\
\hline 5 & 1400 & 1749 & 94,47 & 5,53 & - & 100 & 0,000069 \\
\hline 6 & 1400 & 1739 & 94,69 & 5,31 & - & 100 & 0,000076 \\
\hline 1 & 1400 & 1338 & 100 & - & - & - & 0,000084 \\
\hline 2 & 1400 & 1287 & 100 & - & - & - & 0,000092 \\
\hline
\end{tabular}

A presença do $\mathrm{MgO}$ explica o aumento na temperatura de fusão dessas misturas desfosforantes iniciais. A temperatura de fusão das misturas desfosforantes iniciais preparadas com resíduo de mármore (Misturas $5-8$ ) está acima de $1400^{\circ} \mathrm{C}$. Podese observar que há um aumento na concentração de MgO não dissolvido nessas misturas, que formam fases sólidas com alto ponto de fusão.

A Tabela 5 mostra os resultados dos parâmetros termodinâmicos, via equações empíricas, das escórias desfosforantes finais e os relacionam com o rendimento.

* Contribuição técnica ao 45 Seminário de Aciaria - Internacional, 25 a 28 de maio de 2014, 
Tabela 5. Parâmetros termodinâmicos empíricos das escórias finais com relação ao $\mathrm{P}_{\mathrm{eq}}$ o rendimento

\begin{tabular}{|cccccccccc|}
\hline $\begin{array}{c}\mathbf{N} \\
\text { Escória }\end{array}$ & $\begin{array}{c}\text { Bas. } \\
\text { Final }\end{array}$ & $\mathbf{\Lambda}$ & $\begin{array}{c}\mathbf{L}_{\mathbf{p}} \\
\text { Real }\end{array}$ & $\begin{array}{c}\text { Log }\left(\mathbf{L}_{\mathbf{p}}\right) \\
-\mathbf{H e a l y} \\
{[\mathbf{3}]}\end{array}$ & $\begin{array}{c}\mathbf{L}_{p}- \\
\text { Suito } \\
{[\mathbf{1 3}]}\end{array}$ & $\begin{array}{c}\mathbf{C}_{\mathbf{p}}- \\
\text { Young } \\
{[\mathbf{8}]}\end{array}$ & $\begin{array}{c}\gamma_{P_{2} O_{5}}- \\
\text { Turkdogan } \\
{[\mathbf{1 5}]}\end{array}$ & $\mathbf{P}_{\text {eq. }}$ & \%DeP \\
\hline $\mathbf{5}$ & 2,15 & 0,77 & 1,03 & 3,5283 & 266,13 & $9,11 \mathrm{E}+05$ & $8,96 \mathrm{E}-18$ & 0,000095 & $89 \%$ \\
$\mathbf{1}$ & 1,74 & 0,74 & 0,58 & 3,7552 & 280,28 & $4,22 \mathrm{E}+05$ & $6,36 \mathrm{E}-17$ & 0,000414 & $82 \%$ \\
$\mathbf{7}$ & 2,12 & 0,78 & 0,48 & 3,4186 & 213,00 & $1,70 \mathrm{E}+06$ & $2,99 \mathrm{E}-17$ & 0,000034 & $80 \%$ \\
$\mathbf{6}$ & 1,87 & 0,75 & 0,20 & 2,5166 & 47,62 & $7,44 \mathrm{E}+05$ & $1,02 \mathrm{E}-16$ & 0,000749 & $63 \%$ \\
$\mathbf{8}$ & 1,41 & 0,75 & 0,13 & 2,2155 & 27,91 & $2,93 \mathrm{E}+05$ & $4,65 \mathrm{E}-15$ & 0,000240 & $54 \%$ \\
$\mathbf{4}$ & 1,05 & 0,69 & 0,03 & 0,3446 & 0,32 & $2,89 \mathrm{E}+05$ & $4,50 \mathrm{E}-14$ & 0,070633 & $22 \%$ \\
$\mathbf{3}$ & 1,02 & 0,69 & 0,08 & 0,2940 & 0,25 & $2,14 \mathrm{E}+05$ & $5,89 \mathrm{E}-14$ & 0,093010 & $39 \%$ \\
$\mathbf{2}$ & 1,16 & 0,69 & 0,04 & 0,0992 & 0,21 & $3,52 \mathrm{E}+05$ & $5,83 \mathrm{E}-15$ & 0,094182 & $26 \%$ \\
\hline
\end{tabular}

$\mathrm{Na}$ Tabela 5, o número das escórias finais está relacionado com a ordem decrescente do rendimento. Fazendo análise pelo $P_{\text {eq, }}$ a melhor escória final é a $N^{0} 7$ $\left(\mathrm{RM}+\right.$ Carepa $\left.+\mathrm{Na}_{2} \mathrm{O}\right)$, com menor valor $0,000034 \%$.

Os resultados dos parâmetros termodinâmicos das escórias desfosforantes finais relacionados com o rendimento, estão plotados nas Figuras 4 e 5.

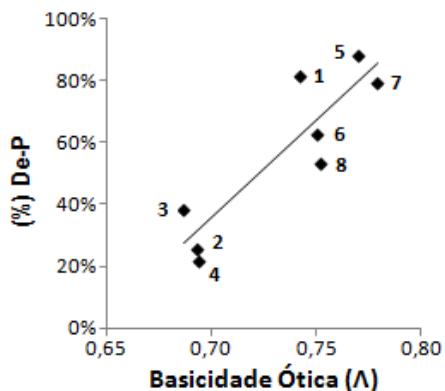

(A)

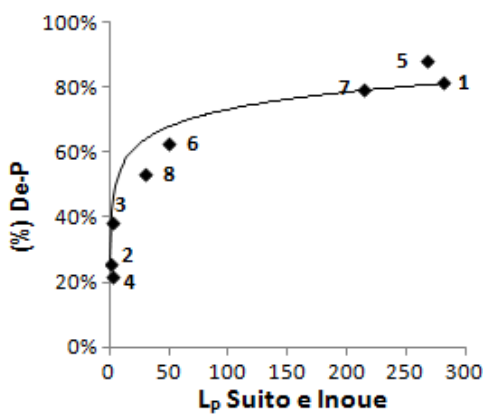

(D)

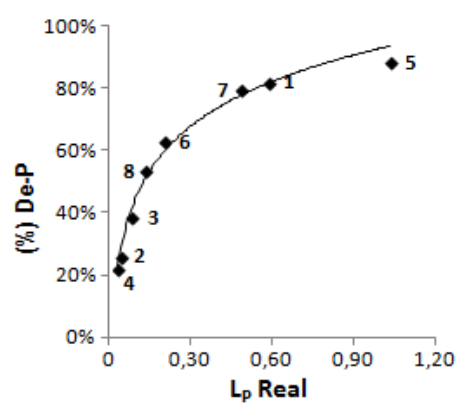

(B)

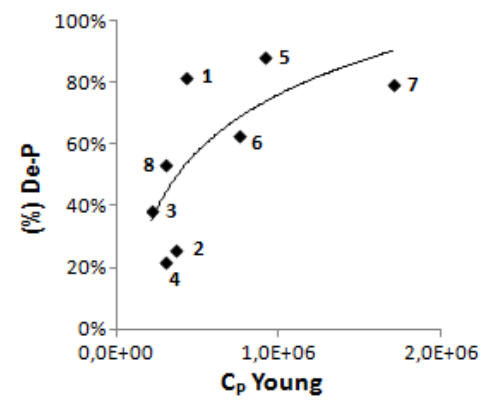

(E)

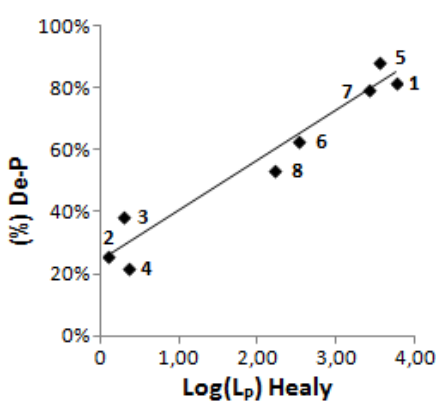

(C)

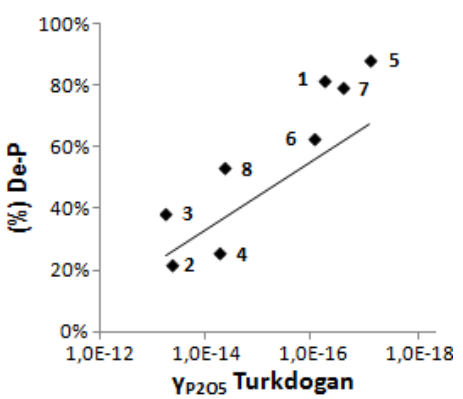

(F)

Figura 4. Relação entre a taxa de De-P e (a) Basicidade ótica; (b) Partição Real Lp, (c) Log(Lp) de Healy [3], (d) Lp de Suito e Inoue [4], (e) Cp de Young [7] e (f) coeficiente de atividade do $\mathrm{P}_{2} \mathrm{O}_{5}$ por Turkdogan [15] das escórias finais.

Nota-se através dos resultados das Figuras 4 e 5 , que os parâmetros termodinâmicos das escórias finais estabelecem uma relação direta com 0 rendimento. Conforme observado, as escórias finais contendo resíduo de mármore foram melhores comparadas com as escórias contendo cal convencional.

A Tabela 6 mostra parâmetros termodinâmicos ( $\left.\mathrm{a}_{\mathrm{CaO}} \mathrm{e} \mathrm{a}_{\mathrm{FeO}}\right)$, calculados pelo programa ThermoCalc, e os parâmetros cinéticos - viscosidades e frações da \% de líquido e \% de sólido, calculados pelo programa FactSage, das escórias finais com relação ao rendimento:

* Contribuição técnica ao 450 Seminário de Aciaria - Internacional, 25 a 28 de maio de 2014, Porto Alegre, RS, Brasil. 


\section{ACJARIA}

Tabela 6. Parâmetros termodinâmicos e cinéticos das escórias finais

\begin{tabular}{|c|c|c|c|c|c|c|c|c|c|c|}
\hline $\mathbf{N}^{\mathbf{0}}$ & $\mathbf{P}_{\mathbf{i}}$ & $\mathbf{P}_{\mathbf{f}}$ & Visc. Liq. & Visc. Efe. & aCaO & aFeO & \%Líq. & \%Sól. & $\mathbf{P}_{\text {eq. }}$ & \%DeP \\
\hline $\mathbf{5}$ & 0,11 & 0,0118 & 0,94 & 2,52 & 0,3128 & 0,3176 & 75,85 & 24,15 & 0,000095 & $89 \%$ \\
\hline $\mathbf{1}$ & 0,11 & 0,0201 & 1,15 & 4,55 & 0,0864 & 0,4047 & 68,66 & 31,34 & 0,000414 & $82 \%$ \\
\hline $\mathbf{7}$ & 0,11 & 0,0220 & 1,20 & 1,31 & 0,2545 & 0,3090 & 79,10 & 21,90 & 0,000034 & $80 \%$ \\
\hline $\mathbf{6}$ & 0,11 & 0,0402 & 1,15 & 2,09 & 0,1201 & 0,2519 & 84,24 & 15,76 & 0,000749 & $63 \%$ \\
\hline $\mathbf{8}$ & 0,11 & 0,0506 & 0,96 & 1,60 & 0,1068 & 0,2151 & 86,33 & 13,67 & 0,000240 & $54 \%$ \\
\hline $\mathbf{3}$ & 0,11 & 0,0672 & 3,32 & 3,68 & 0,0522 & 0,0049 & 97,05 & 2,95 & 0,070633 & $39 \%$ \\
\hline $\mathbf{2}$ & 0,11 & 0,0810 & 2,41 & 3,82 & 0,0444 & 0,0195 & 95,56 & 4,44 & 0,093010 & $26 \%$ \\
\hline $\mathbf{4}$ & 0,11 & 0,0855 & 2,14 & 2,76 & 0,0472 & 0,0287 & 92,79 & 7,21 & 0,094182 & $22 \%$ \\
\hline
\end{tabular}

Pela Tabela 6, através da termodinâmica computacional, nota-se que a escória final com melhor eficiência de desfosforação foi à do experimento $N^{0} 5(R M+C a r e p a)$, sem o uso dos fundentes $\mathrm{CaF}_{2}$ e $\mathrm{Na}_{2} \mathrm{O}$, que combina os melhores parâmetros termodinâmicos e cinéticos da sua escória final, obtendo $P_{\text {eq }}$ de $0,000095 \%$ e melhor eficiência de desfosforação com rendimento de $89 \%$. Os gráficos a seguir, plotam os resultados da Tabela 6 com relação ao rendimento.

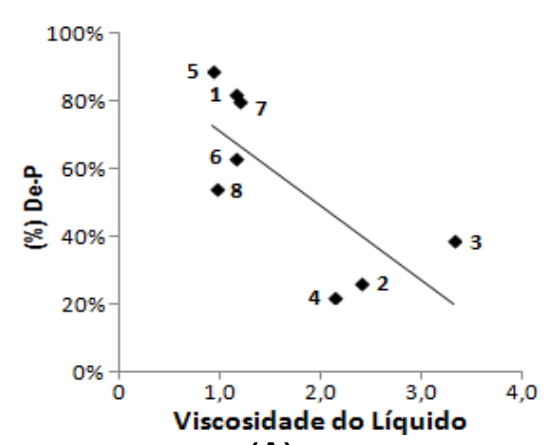

(A)

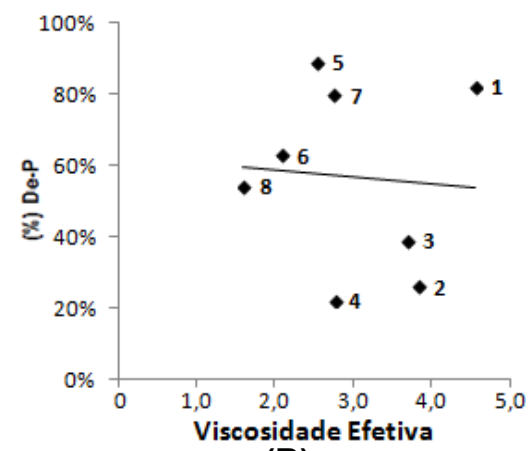

(B)

Figura 5. Viscosidade da (a) fração liquida e (b) fração sólida das escórias finais e o rendimento.

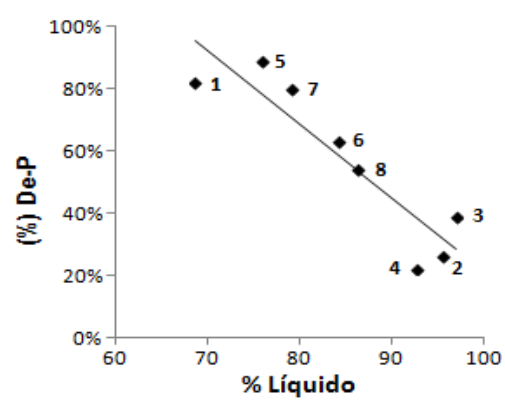

(A)

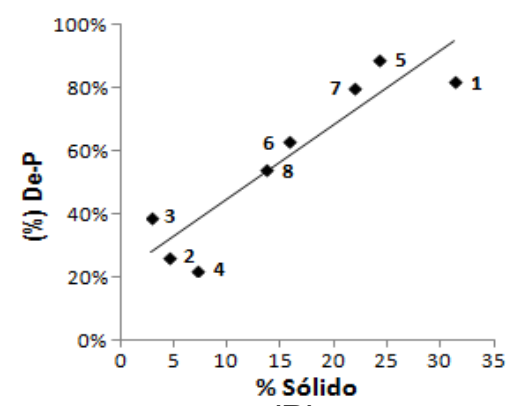

(B)

Figura 6. Relação entre a (a) \% de líquido e (b) \% de sólido das escórias finais e o rendimento.

Conforme mostrado nas figuras acima, analisando os parâmetros da \% de líquido e da $\%$ de sólido, Figura 6 , respectivamente, quanto menor a viscosidade das escórias finais, maior o rendimento.

Os gráficos na Figura 7, plotam os resultados do rendimento com relação à composição química das escórias finais.

\footnotetext{
* Contribuição técnica ao 450 Seminário de Aciaria - Internacional, 25 a 28 de maio de 2014,
} 


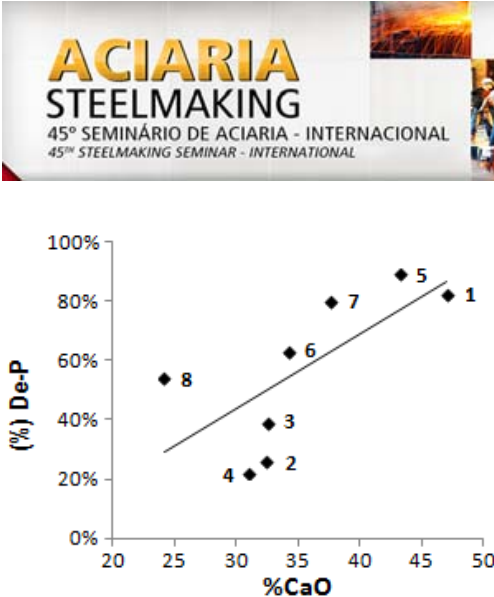

(A)

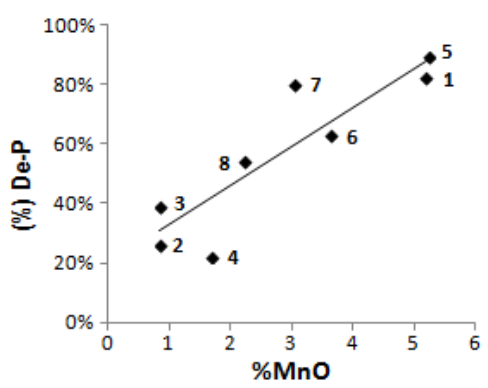

(D)

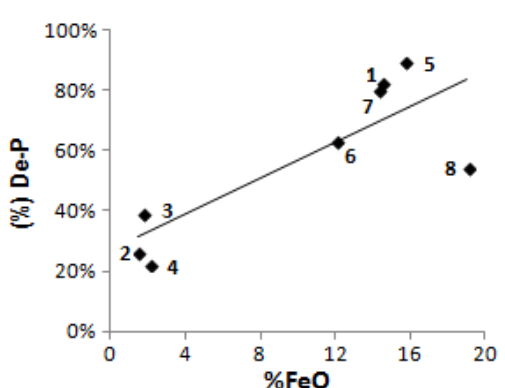

(B)

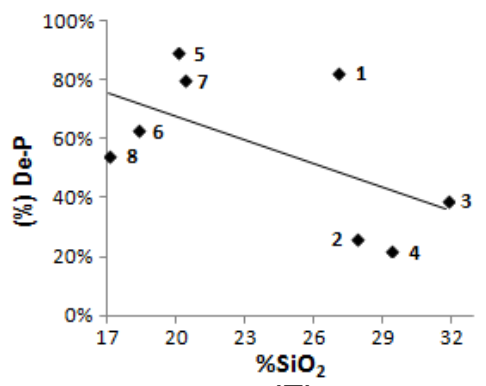

(E)

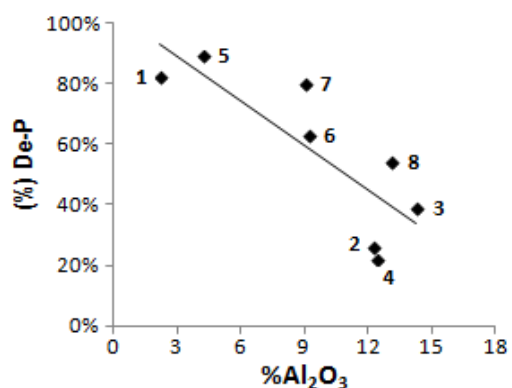

(C)

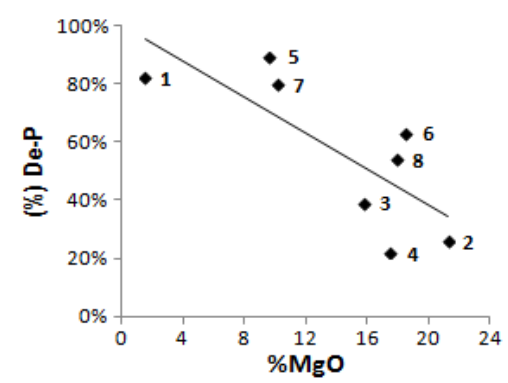

(F)

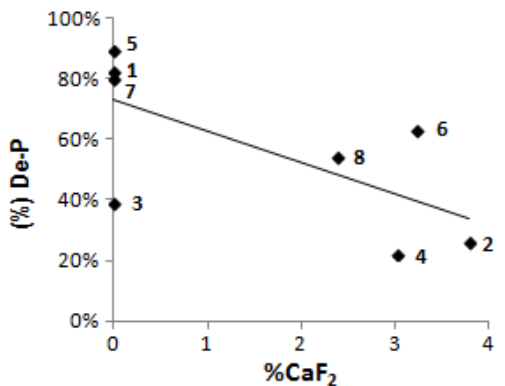

(G)

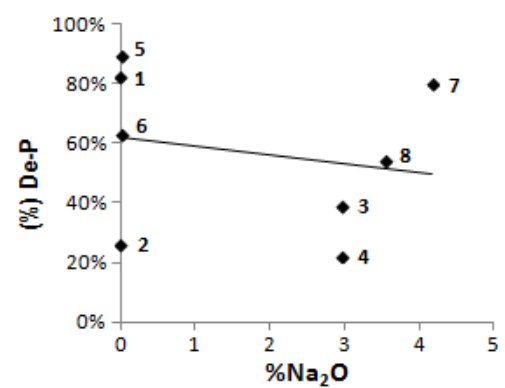

(H)

Figura 7. Relação entre o teor de (a) $\mathrm{CaO}$, (b) $\mathrm{FeO}$, (c) $\mathrm{Al}_{2} \mathrm{O}_{3}$, (d) $\mathrm{MnO}$, (e) $\mathrm{SiO}_{2}$, (f) $\mathrm{MgO}$, (g) $\mathrm{CaF}_{2}$ e (f) $\mathrm{Na}_{2} \mathrm{O}$ das escórias finais e o rendimento.

Conforme resultado mostrado nas figuras acima, houve uma relação direta entre a composição química das escórias finais e o rendimento. Conforme observado nas Figuras $7 \mathrm{a}$ e $7 \mathrm{~b}$, quanto maior a $\% \mathrm{CaO}$ e $\% \mathrm{FeO}$ nas escórias finais, respectivamente, maior o rendimento. As escórias finais com resíduo de mármore possuem maior \% $\mathrm{CaO}$ e \%FeO, comparado com a cal convencional. Quanto maior a $\% \mathrm{CaO}$ e a \%FeO nas escórias finais, maior $\mathrm{a}_{\mathrm{CaO}}$ e $\mathrm{a}_{\mathrm{FeO}}$, respectivamente, conforme os parâmetros termodinâmicos calculados pelo programa FactSage. Esses resultados estão de acordo com Ishii e Fruehan [16], pois a partir dos resultados da análise química das escórias finais, $0 L_{p}$ estava diretamente relacionado com a $\% \mathrm{CaO}$ e \%FeO. As escórias finais com resíduo de mármore possuem menores $\% \mathrm{SiO}_{2}$ e $\% \mathrm{MgO}$, comparado com a cal convencional. Maiores teores de $\mathrm{SiO}_{2}$ e $\mathrm{MgO}$ na escória diminuem a basicidade e aumentam a viscosidade da escória, respectivamente, o que prejudica o processo de desfosforação [14]. A Figura $7 \mathrm{~g} \mathrm{e}$ (H) resume a variação da $\% \mathrm{CaF}_{2}$ e $\% \mathrm{Na}_{2} \mathrm{O}$ das escórias finais, respectivamente, com relação ao rendimento. Conforme observado, quanto menor a $\% \mathrm{CaF}_{2}$ e a $\% \mathrm{Na}_{2} \mathrm{O}$, maior o rendimento da desfosforação. As escórias finais com resíduo de mármore possuem menores $\% \mathrm{CaF}_{2}$ e $\% \mathrm{Na}_{2} \mathrm{O}$, comparado com a cal convencional. A presença dos fundentes $\% \mathrm{CaF}_{2}$ e $\% \mathrm{Na}_{2} \mathrm{O}$ nas escórias mudaram completamente a composição química da escória, devido a contaminação com $\mathrm{Al}_{2} \mathrm{O}_{3}$ e $\mathrm{MgO}$, o que prejudica o processo de desfosforação [17]. E diminuem a atividade do $\mathrm{CaO}$.

* Contribuição técnica ao $45^{\circ}$ Seminário de Aciaria - Internacional, 25 a 28 de maio de 2014, Porto Alegre, RS, Brasil. 
Como as escórias 5 e 1 não tiveram contaminação do cadinho e nem do tubo de alumina, e melhores resultados na De-P. Mantendo suas características originais, estas foram analisadas separadamente

A Figura 8 mostra a característica miscroestrutural da escória final $N^{0} 1$ (contendo cal) e $N^{\circ} 5$ (contendo resíduo de mármore), respectivamente:
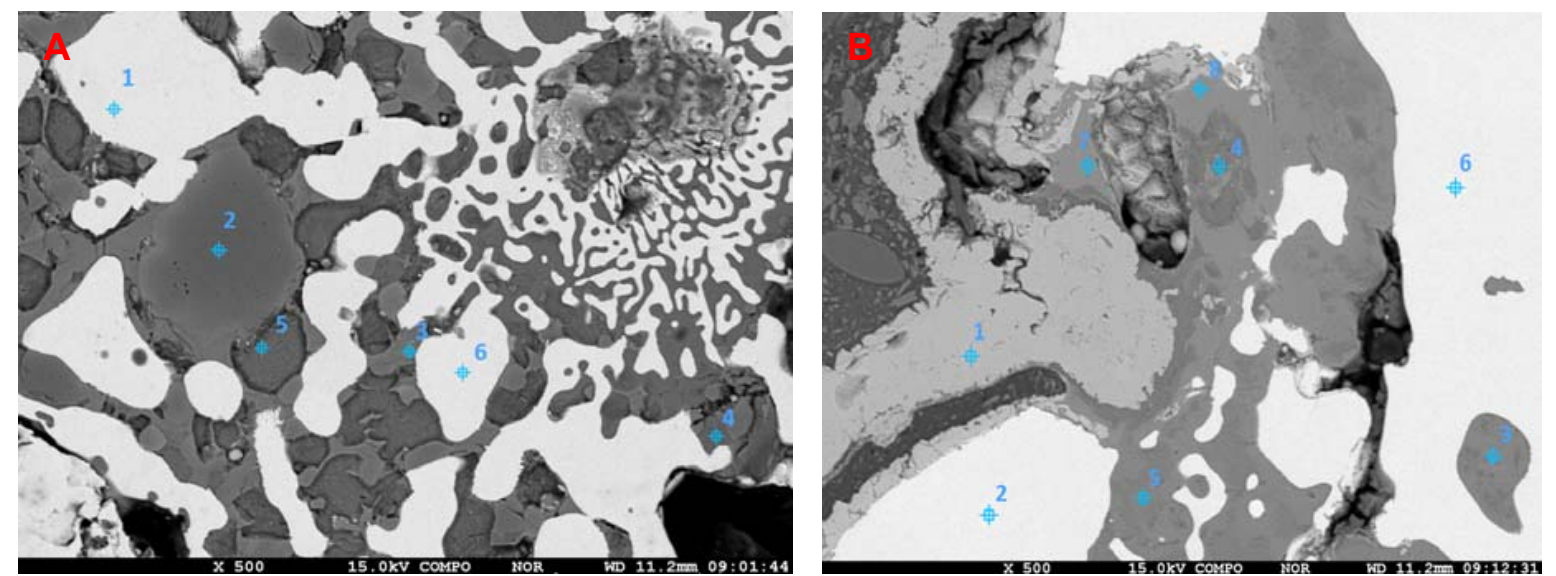

Figura 8. Imagens do EDS: (a) da escória № 1 com cal convencional: 500X e (b) da escória № 5 com resíduo de mármore: 500X.

Os pontos nas Figuras $8 \mathrm{a}$ e $8 \mathrm{~b}$ foram analisados. O resultado revelou a presença dos elementos cálcio ( $\mathrm{Ca})$, silício ( $\mathrm{Si})$, magnésio $(\mathrm{Mg})$, manganês $(\mathrm{Mn})$, alumínio (Al), ferro (Fe) e oxigênio (O). Os dados no ponto 3 não foram analisados

Os pontos 4 e 5 da Figura $8 b$ foram identificadas como silicato dicálcio $\left(2 \mathrm{CaO} . \mathrm{SiO}_{2}\right)$, onde verifica-se que o $\mathrm{Ca}$ e o $\mathrm{Si}$ coexistem na mesma fase. Os resultados das análises do EDS confirmam a presença do fósforo, na forma de solução sólida $3 \mathrm{CaO} . \mathrm{P}_{2} \mathrm{O}_{5}$, dissolvido na fase $2 \mathrm{CaO} . \mathrm{SiO}_{2}$.

Esses pontos mostram a formação de uma escória adequada para a reação de desfosforação, sendo capaz de absorver e reter o fósforo em sua estrutura. $O$ fósforo na forma de fosfato tricálcio $\left(3 \mathrm{CaO} \cdot \mathrm{P}_{2} \mathrm{O}_{5}\right)$ encontra-se em solução sólida com o silicato dicálcio (2CaO. $\left.\mathrm{SiO}_{2}\right)$, conforme a proporção dos elementos encontrados no EDS, da escória final No 5 (RM + Carepa) e $N^{0} 1$ (Cal + Carepa), respectivamente.

\section{CONCLUSÃO}

Diante dos resultados conseguidos nos experimentos de desfosforação realizados com misturas desfosforantes do sistema $\mathrm{CaO}-\mathrm{FeO}-\mathrm{SiO}_{2}$, contendo cal convencional e resíduo de mármore, temos que:

- O aquecimento das misturas desfosforantes iniciais mostrou que as escórias contendo o resíduo de mármore possuem elevada temperatura final de fusão, ao contrário das misturas contendo a cal convencional, que a $1400^{\circ} \mathrm{C}$ estavam 100\% líquidas, não havendo \% de sólido;

- As escórias finais apresentaram uma relação direta entre os parâmetros termodinâmicos empíricos e os rendimentos de desfosforação, sendo que as escórias contendo o resíduo alcançaram maiores taxas de desfosforação;

- Exceto as escórias $N^{0} 1$ (Cal + Carepa) e $N^{\circ} 5$ (RM + Carepa), as demais tiveram sua composição química alterada, devido a contaminação com MgO e $\mathrm{Al}_{2} \mathrm{O}_{3}$, proveniente do ataque químico dessas escórias com o cadinho de $\mathrm{MgO}$ e o impeller de $\mathrm{Al}_{2} \mathrm{O}_{3}$. $\mathrm{O}$ ataque foi proveniente pela presença de $\mathrm{CaF}_{2} \mathrm{e}$ $\mathrm{Na}_{2} \mathrm{O}$, principalmente nas escórias contendo cal convencional;

* Contribuição técnica ao 450 Seminário de Aciaria - Internacional, 25 a 28 de maio de 2014, 
- As escórias contendo resíduo de mármore apresentaram menores \% de líquido e maiores \% de sólido, o que diminui a atividade do $\mathrm{P}_{2} \mathrm{O}_{5}$ na fase líquida da escória final, pois fases sólidas de $2 \mathrm{CaO}^{-\mathrm{SiO}_{2}}$ e $3 \mathrm{CaO} \cdot \mathrm{P}_{2} \mathrm{O}_{5}$ formam solução sólida $2 \mathrm{CaO} \cdot \mathrm{SiO}_{2}-3 \mathrm{CaO} \cdot \mathrm{P}_{2} \mathrm{O}_{5}$;

- As escórias № 1 (Cal + Carepa) e No 5 (RM + Carepa), com rendimentos de desfosforação de 82 e $89 \%$ respectivamente, possuíram valores muito próximos de atividade do $\mathrm{CaO}$ e $\mathrm{FeO}$, além de viscosidade da \% de líquido. $\mathrm{O}$ que provavelmente aumentou a eficiência do resíduo de mármore foi a presença de fases sólidas e a de carbonatos, que aumentaram a cinética da reação de desfosforação;

- As análises de MEV mostraram mais $\% \mathrm{P}_{2} \mathrm{O}_{5}$ presente na escória $\mathrm{N}^{0} 5$ comparado com a escória $N^{0} 1$, o que pode ser fator importante para se aproveitar a presença de fases sólidas em escórias de desfosforação.

\section{REFERÊNCIAS}

1 Stubbles JR. Phosphorus and sulfur in steelmaking I: the origin of phosphorus and sulfur - Part I. Iron and Steelmaker. 1986;1:34-35.

2 Campos VF. Tecnologia de fabricação do aço líquido - Fundamentos. $3^{\mathrm{a}}$ ed. Belo Horizonte: UFMG; 1985. p.141-163.

3 Borode JO. Phosphorus partition ratio and phosphate capacity of $\mathrm{Na} 2 \mathrm{O}-\mathrm{SiO} 2$ at $1,400^{\circ} \mathrm{C}$. Ironmaking and Steelmaking.1998;25(2):144-149.

4 Suito $\mathrm{H}$, Inoue R. Behavior of phosphorous transfer from CaO-FetO-P2O5(-SiO2) slag to CaO Particles. ISIJ International. 2006;46(2):180-187.

5 Healy G. A New look at phosphorus distributuion. Journal of the Iron and Steel Institute. 1970;208:664-668.

6 Wagner C. The concept of basicity of slags. Metallurgical Transactions B. 1975;6B:405409.

7 Young RW. Use of the optical basicity concept for determining phosphorus and sulphur slag/metals partitions. Technical Steel Research Steelmaking. 1991;1:8-104.

8 Campos VF. Curso Sobre Pré-Refino de Ferro-Gusa. $1^{\text {a }}$ ed. Belo Horizonte: Universidade Federal de Minas Gerais; 1984. p. 31.

9 Koros $P$. The making, shaping and treating of steel: steelmaking and refining. Charpter 7: Pretreatment of Hot Metal. Pittsburg: The AISE Steel Foundation; 1998.

10 Kitamura SY, Yonezawa K, Ogawa Y, Sasaki N. Improvement of reaction efficiency in hot metal dephosphorization. Ironmaking and Steelmaking. 2002;29(2):121-124.

11 Pahlevani F, Kitamura S, Shibata $\mathrm{H}$, Maruoka N. Distribution of $\mathrm{P}_{2} \mathrm{O}_{5}$ between Solid Solution of $2 \mathrm{CaO} . \mathrm{SiO}_{2}-3 \mathrm{CaO} . \mathrm{P}_{2} \mathrm{O}_{5}$ and liquid phase. ISIJ International. 2010;50(6):822829.

12 Ito K, Terasawa M. Utilization of multiphase fluxes for the dephosphorization of hot metal. Process Metallurgy. Steel Research int. 2009;80:733-736.

13 Suito $\mathrm{H}$, Inoue R. Phosphorus partition between $2 \mathrm{CaO} . \mathrm{SiO}_{2}$ particles and $\mathrm{CaO}-\mathrm{FeO}-\mathrm{SiO}_{2}$ slags. ISIJ International. 2006;46(2):174-179.

14 Jung S, Min D, Rhee C. Solubility of MgO new ironmaking process slags. ISIJ International. 2007;47(12):1718-22.

15 Turkdogan ET, Pearson J. Activities of constituintes of iron and steelmaking slags: iron oxide. Journal of the Iron and Steel Institute. 1953;175(23):173-393.

16 Ishii H, Fruehan RJ. Dephosphorization equilibria between liquid iron and highly basic CaO-based slags saturated with MgO. Metallurgical and Materials Transactions B. 1997; 24B:47-54.

17 Kunisada $\mathrm{K}$, Iwai $\mathrm{H}$. Effect of $\mathrm{Na}_{2} \mathrm{O}$ on phosphorus distribution between liquid iron and CaO-based slags. Transactions ISIJ. 1987;27:263-269.

* Contribuição técnica ao 45 Seminário de Aciaria - Internacional, 25 a 28 de maio de 2014, 